\title{
e-Phaïstos
}

e-Phaïstos

Revue d'histoire des techniques / Journal of the history

of technology

II-2 | 2013

Les sources de l'histoire des techniques (2)

\section{Les brevets d'invention et leurs dessins techniques}

A propos de la conservation du patrimoine de la création technique

Patents and Their Technical Drawings

\section{Gérard Emptoz}

\section{CpenEdition}

1 Journals

Édition électronique

URL : http://journals.openedition.org/ephaistos/7145

DOI : 10.4000/ephaistos. 7145

ISSN : 2552-0741

Éditeur

IHMC - Institut d'histoire moderne et contemporaine (UMR 8066)

Édition imprimée

Date de publication : 15 décembre 2013

Pagination : 43-55

ISSN : 2262-7340

\section{Référence électronique}

Gérard Emptoz, "Les brevets d'invention et leurs dessins techniques », e-Phaïstos [En ligne], II-2 | 2013,

mis en ligne le 12 décembre 2019, consulté le 14 décembre 2019. URL : http://

journals.openedition.org/ephaistos/7145; DOI : 10.4000/ephaistos.7145 


\section{Les brevets d'invention et leurs dessins techniques}

\section{À propos de la conservation du patrimoine de la création technique ${ }^{1}$}

Les archives de l'invention sont constituées, tout au moins une partie, par celles qui relèvent des dépôts faits en vue d'une protection de la propriété industrielle. Ce domaine particulier recouvre, comme on le sait, la protection de l'invention par les brevets, la protection des dessins et des modèles industriels, ainsi que celle des marques de fabrique. Ces documents émanent directement des acteurs de la création technique (inventeurs, industriels, créateurs) au cours de l'histoire industrielle de notre pays. C'est ainsi une partie importante du patrimoine immatériel des industries qui se trouve présente dans ces archives. Leur intérêt est d'autant plus grand que bien souvent elles constituent les rares traces laissées par d'innombrables inventeurs à l'origine d'innovations remarquables, et par de nombreuses petites ou moyennes entreprises, dont la durée de vie a été souvent relativement courte mais dont l'apport technologique a pu être important. Des études menées par des historiens sur les fonds de brevets d'invention ont montré le grand intérêt présenté par ces archives pour reconstituer l'histoire de l'innovation². Accéder à cette mémoire constituée par les archives de la propriété industrielle, c'est pouvoir comprendre la dynamique des innovations, retrouver les racines de la création et

\author{
Gérard Emptoz \\ Centre Françoise Viète \\ Université de Nantes
}

les moyens destinés à attirer l'attention des éventuels consommateurs.

\section{Aperçu des différentes institutions qui ont enregistré les dépôts de la propriété indus- trielle}

Les événements de l'histoire de la propriété industrielle en France ont abouti au fait qu'une partie importante de ces fonds est actuellement conservée par l'Institut national de la propriété industrielle (INPI), établissement public doté de la personnalité civile et de l'autonomie financière, placé auprès du ministère en charge de l'industrie.

L'histoire de la propriété industrielle commence véritablement en France durant la Révolution avec les premières lois sur les brevets d'invention ( 7 janvier - 25 mai 1791), qui crée le Directoire des brevets d'invention, service placé auprès du ministère de l'intérieur de l'époque3. Le 17 Vendémiaire an VII (8 octobre 1798), le Directoire exécutif charge le Conservatoire des Arts et Métiers de recevoir le dépôt des originaux des brevets expirés et, conformément à ses directives, le ministre de l'intérieur charge le Conservatoire de publier les brevets d'invention.

Tout au long du XIX ${ }^{\mathrm{e}}$ siècle, le service en charge de l'enregistrement des brevets d'invention, puis des marques de fabrique à partir de la loi du 27 juin 1857, dépend tour à tour du ministère de l'intérieur 
et de celui du commerce. En 1882, est créé le bureau de la propriété industrielle, rattaché à la Direction de l'industrie du ministère du Commerce et de l'industrie4.

C'est en 1901 (loi et décret du 9 juillet) que l' «Office national des brevets d'invention et des marques de fabrique » est créé. Cet office, rebaptisé "Office national de la propriété industrielle » (ONPI) en 1902, s'installe en 1919 dans l'immeuble situé au 26 bis, rue de Petrograd, aujourd'hui rue de Saint-Pétersbourg. Depuis 2013, il se trouve à une nouvelle adresses.

L' «Institut national de la propriété industrielle » (INPI) est créé par la loi du 19 avril 1951. Il a pour mission de participer à l'élaboration du droit de la propriété industrielle, de recevoir les dépôts et délivrer les titres de propriété industrielle (brevets, marques, dessins et modèles), de mettre à disposition du public les informations officielles dans le domaine de la propriété industrielle, de tenir le registre national du commerce et des sociétés, ainsi que le répertoire central des métiers ${ }^{6}$.

C'est pourquoi, en tant qu'héritier des fonds des différentes institutions qui se sont succédées depuis 1791, et qui viennent d'être évoquées, l'INPI aujourd'hui conserve dans ses locaux un fonds d'archives unique, constitué par l'intégralité des brevets d'invention déposés depuis 1791, des marques depuis 1858 et des dessins et modèles depuis 1910, représentant plus de $150 \mathrm{~km}$ de documents originaux 7 .

\section{Les archives des brevets d'invention}

«Sont brevetables les inventions nouvelles impliquant une activité inventive et susceptibles d'application industrielle ${ }^{8}$. La nouveauté d'une invention s'apprécie au regard de l'état de la technique, qui « est constitué par tout ce qui a été rendu accessible au public avant la date de dépôt de la demande de brevet par une description écrite ou orale, un usage ou tout autre moyen $» 9$.

Les lois de 1791 reconnaissent et garantissent la propriété des inventeurs; le brevet d'invention est établi comme un contrat d'une durée limitée, qui permet au déposant de bénéficier d'un monopole pour exploiter son brevet; à la fin de la durée de protection, l'invention fait partie du domaine public. Trois durées de protection sont proposées aux inventeurs, qui doivent faire leur choix au moment du dépôt : cinq, dix ou quinze ans. Bien que révisée en 1844, ces dispositions restèrent inchangées jusqu'en 1939, lorsque la durée de validité d'un brevet a été portée à vingt ans. Le maintien de la protection d'une année à l'autre dépend du paiement d'une taxe annuelle, principe encore en vigueur aujourd'hui. En 1968, une nouvelle loi est adoptée sur les brevets d'invention, pour introduire les mesures validées par les pays membres du Conseil de l'Europe. Depuis 1992, cette loi fait partie d'un ensemble législatif couvrant tous les domaines de la propriété industrielle ${ }^{10}$.

Dès l'origine et jusqu'en 1901, le déposant d'un brevet devait remettre un paquet cacheté contenant les pièces suivantes : une lettre adressée au ministre de l'Intérieur ${ }^{11}$, dans laquelle le déposant demande la délivrance d'un brevet; un mémoire descriptif, dans lequel le déposant explique de façon détaillée les «principes, moyens et procédés » qui constituent son invention; des dessins, remis en double exemplaire (plans, coupes et élévations) ou bien un modèle de l'invention. Jusqu'en 1844 les documents ont été soumis au Comité consultatif des Arts et Manufactures pour avis sur l'accomplissement et l'exactitude des formalités décrites ci-dessus. C'est ainsi que les brevets sont délivrés avec la mention suivante : « le gouvernement, en accordant un brevet d'invention, n'entend garantir en aucune manière, ni la priorité, ni le mérite, ni le succès d'une invention $»^{12}$.

Un catalogue, recensant tous les brevets déposés, est édité chaque année par le ministère. Toute personne peut demander à les consulter avant de déposer un brevet, afin de vérifier si l'invention est véritablement nouvelle. A partir de 1883 , sont publiées des listes hebdomadaires dans chaque numéro du Bulletin officiel de la propriété industrielle 


\section{$(\mathrm{BOPI})^{13}$.}

Lorsque la validité d'un brevet prend fin, ou bien lorsqu'il a été déchu de ses droits, il a donc été publié par le Conservatoire des arts et métiers, à moins que le déposant obtienne une prolongation ou le secret de son invention. Cette collection éditée par le Conservatoire, et intitulée Description des machines et procédés, est la seule publication des brevets pour le XIX ${ }^{\mathrm{e}}$ siècle, mais elle n'est ni intégrale, ni systématique. Les textes des brevets sont en effet corrigés, résumés, en totalité ou en partie, regroupés (par exemple, le brevet initial avec les additions), abrégés, et parfois seules les données bibliographiques sont indiquées. Les planches des dessins sont renvoyées à la fin de chaque volume.

Lorsque les brevets sont imprimés, c'est à dire à partir de 1902, l'accès à ces documents est naturellement simplifié. Actuellement, l'accès peut être fait par Internet (voir plus loin).

Tous les brevets déposés en France sont conservés par l'INPI'14.

\section{L'accès au contenu intégral des brevets : le Guide des archives de la propriété indus- trielle et les bases informatisées de l'INPI}

Il résulte de l'évolution des modalités d'organisation des documents que les outils d'accès aux brevets sont différents selon les périodes concernées.

Pour la période allant de 1791 à 1902 , tous les brevets sont des manuscrits ou des tapuscrits selon la période. Les dossiers originaux conservés comportent une série d'informations sur l'inventeur, la description de l'invention, ainsi que les planches de dessin d'accompagnement. Il est ainsi possible de faire des recherches par noms de déposants et par matières de plusieurs façons. La consultation des Catalogues des brevets d'invention, année après année, jusqu'en 1883, puis, à partir des années suivantes, dans la collection des volumes du Bulletin officiel de la propriété industrielle constitue la méthode classique. Ces recherches sont souvent longues, en particulier parce que les tables des catalogues sont construites à partir du langage technique de l'époque et font usage de mots ou de néologismes difficiles à saisir de nos jours. Actuellement l'INPI a mis en ligne l'intégralité des brevets déposés entre 1791 et 1855 , ce qui rend la recherche très efficace et très rapide pour cette période historique ${ }^{15}$. L'accès sera progressivement étendu jusqu'à l'année $1901^{16}$. La base des brevets du XIXe siècle ${ }^{17}$ permet des recherches avec des possibilités étendues d'interrogations. Une série de mots-clés accompagne chaque brevet. La recherche par nom d'inventeur est aussi très facilitée par la structure des fiches descriptives de chaque document. Enfin, les reproductions issues de photographies des dessins contenus dans les brevets comportent une échelle qui permet de reconstituer les documents aux dimensions d'origine.

Pour les brevets du $\mathrm{XX}^{\mathrm{e}}$ siècle, le fonds est à la fois accessible grâce à des tables par déposants et par matières publiés dans le Bulletin officiel de la propriété industrielle (BOPI), mais aussi par Internet $^{18}$. Toutefois les classements ayant varié au cours du temps, l'accès n'est pas très aisé dans certains cas.

On peut signaler aussi qu'à partir de 1902, les textes originaux des brevets et les correspondances des inventeurs accompagnant les dépôts ont été légalement détruits au bout de trente ans. Il ne reste donc comme archives que la version imprimée des brevets. Cette disposition de destruction légale a été revue, et la conservation des correspondances a été reprise à partir de l'année $1968^{19}$.

Pour les historiens des techniques et de l'innovation, l'ensemble de ces archives et de cette documentation sur les brevets permet un accès quasiment direct aux inventeurs pour l'étude des modalités de la création technique.

Par ailleurs, dans le cadre des actions engagées pour rendre plus accessibles l'ensemble des archives des brevets d'invention, et plus généralement pour la mise en valeur de l'ensemble de son patrimoine, la cellule Archives et Patrimoine de l'INPI, 
créée en 1997, a réalisé un guide destiné à faciliter la consultation et l'accès aux documents. En 2002, l'Institut a publié l'ouvrage sous le titre Aux sources de la propriété industrielle, Guide des archives de la propriété industrielle, ouvrage destiné à tous les chercheurs intéressés par ces archives d'un intérêt historique exceptionnel ${ }^{20}$. Ce travail a nécessité la réunion d'une abondante documentation, non seulement sur les brevets, mais aussi sur le fonds des «Dessins et modèles », ainsi que celui des "Marques déposées ».

Comportant des études historiques et abondamment illustré, l'ouvrage donne sur chacun de ces domaines des moyens d'accès aux informations recherchées et tous les détails nécessaires à faciliter les recherches ${ }^{21}$. Il est destiné à aider à retrouver tous documents relatifs à la propriété industrielle en France.

\section{Dessin technique et brevets d'invention en France aux XIXème et XXème siècles : quelques repères ${ }^{22}$}

Les dessins sont fréquemment rencontrés dans les documents descriptifs des inventions. Quelques exemples de dessin extraits de brevets déposés entre le début du XIX ${ }^{\mathrm{e}}$ siècle et la période contemporaine viennent illustrer une facette peu étudiée de la représentation des objets inventés. Pour la replacer dans une perspective plus large, il sera nécessaire de l'inscrire dans une histoire générale du dessin technique. Étudiée par différents auteurs, dont on citera plus loin quelques travaux, cette histoire pourra éclairer des aspects évoqués dans le présent article.

Au préalable il faut remarquer que les brevets ne comportent pas tous nécessairement un ou plusieurs dessins. Il n'y a d'ailleurs pas d'obligation en

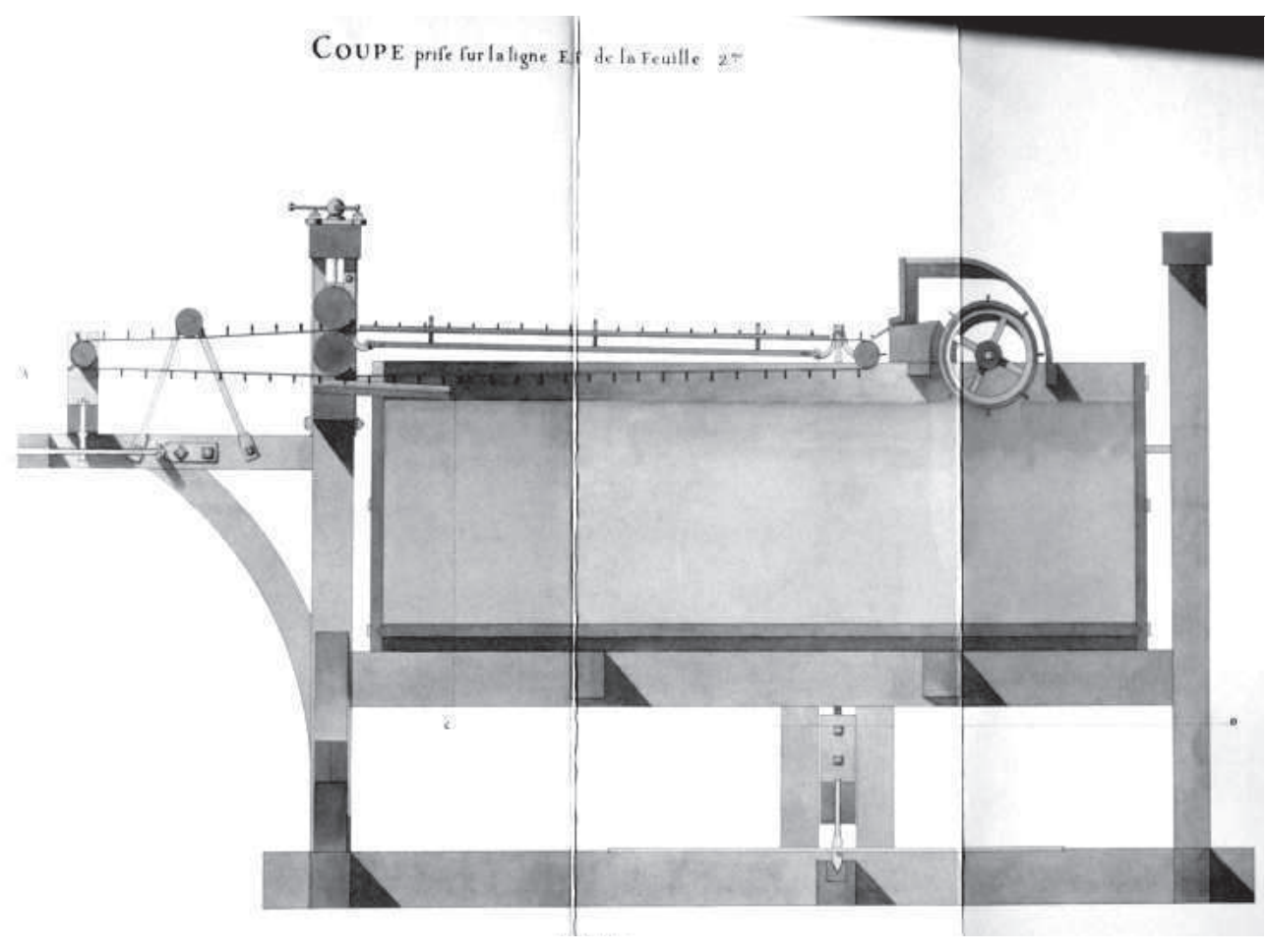

Figure 1 : Le dessin technique des brevets encore à la recherche de sa voie : un dessin d'architecte à la fin du XVIII ${ }^{e}$ siècle. Vue en coupe de la machine à fabriquer le papier d'une très grande étendue, par L. N. Robert (18-O11799). Extraits de brevets (Archives de l'INPI). 
ce domaine. En effet de nombreuses inventions ne correspondent pas nécessairement à des machines, ou à des éléments de machine et d'autres dispositifs techniques particuliers. Les brevets peuvent en effet porter sur des procédés, ou proposer de nouvelles techniques, et, ainsi, ne pas décrire une réalisation concrète.

Dans le cadre de cet article, le choix se limitera volontairement à montrer l'évolution du dessin technique au cours du temps.

Plusieurs remarques peuvent être faites au sujet des dessins contenus dans les brevets d'invention. Les illustrations qui vont suivre permettent de les faire apparaître.

La machine à papier de L.N. Robert servira ici de point de départ. Déposé en 1799, le dessin est très similaire à ceux de son époque (figure 1). C'est une représentation classique, aquarellée, dessin aux traits fins, avec des ombres portées, qui relève du dessin d'architecte du XVIII ${ }^{\mathrm{e}}$ siècle. La représentation de l'objet inventé, une machine, est donc très classique. La loi de 1791 vient d'être promulguée depuis huit ans, et le nombre de dépôts est encore très limité. Il n'existe pas encore de culture particulière en matière de brevet et ce dessin de Robert est représentatif des dessins de la même période historique.

Des évolutions peuvent être constatées dans le graphisme des

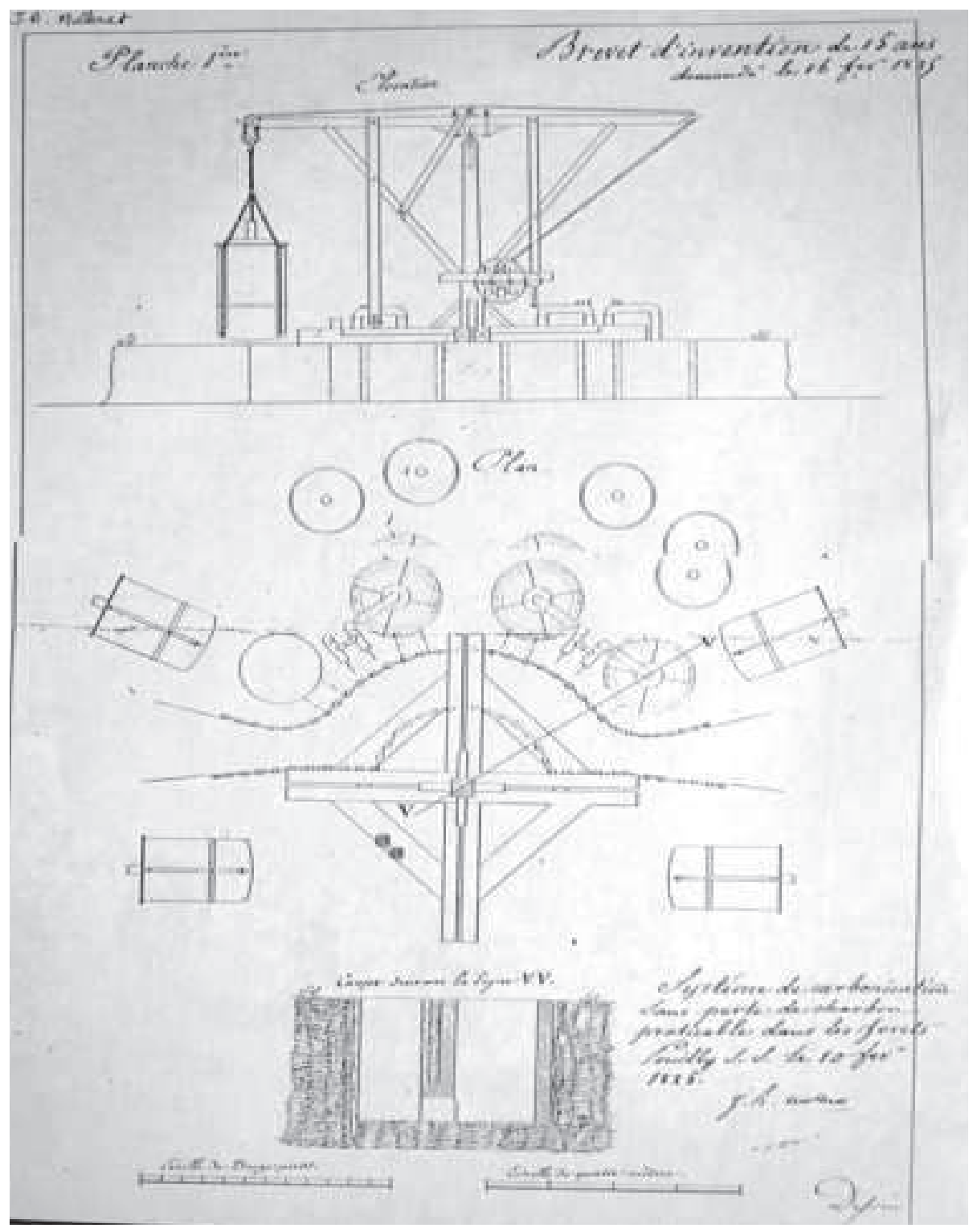

Figure 2 : Le trait devient progressivement très simplifié, pas de couleurs, pas de dimensions, des légendes sommaires. Ensemble complet, plan et coupe, pour le brevet d'un «Système de carbonisation sans perte de charbon praticable dans les forêts » par J. B. Mollerat (10-03-1825). Extraits de brevets (Archives de l'INPI). 
brevets, principalement au cours du XIXe siècle. Rappelons que jusqu'en 1901, les brevets ne sont pas imprimés. En effet, malgré plusieurs tentatives en vue de normaliser les dossiers, notamment comme c'était le cas en Grande-Bretagne, la présentation des dossiers est restée libre. Les documents de toute la période sont de toutes dimensions, et leurs contenus sont multiformes. Les dimensions des dessins ne sont soumises à aucune règle. Dans ce contexte, c'est naturellement une pénétration progressive de représentations normées qui va émerger à mesure que des normes sont diffusées et enseignées dans les écoles et les instituts techniques.

La figure 2 signale une première évolution. Le brevet a été déposé par un grand industriel bourguignon, J-B. Mollerat, personnage très au fait des techniques les plus avancées de son époque ${ }^{23}$. A partir de la lecture des dessins, il est possible de les relier à la culture technique des inventeurs. Lorsque le graphisme est bien de la main même de l'inventeur, cela peut être utile, par exemple, lorsque la profession de l'inventeur n'est pas mentionnée dans le dossier du brevet. Le brevet Mollerat est celui d'un chimiste, homme d'affaires, qui a initié la première chimie du bois et ses sous-produits obtenus par distillation, et qui connaissait bien le monde anglais et ses productions, notamment au sujet des combustibles. Le dessin de ce brevet déposé en 1825 signale une évolution du graphisme, tendant vers la simplicité, l'absence de volume, un à-plat très technique, sans détails superflus. À cette époque, le dessin technique suit de nouvelles règles, comme l'a souligné Yves Deforges²4.

Au milieu du XIX ${ }^{\mathrm{e}}$ siècle, le dessin d'un des brevets de Louis Pasteur (figure 3), déposé en 1871 « pour un nouveau procédé pour la fabrication de la bière ", permet de poser la question de savoir si le dessin est de la main du savant, d'un de ses collègues, ou d'un aide du laboratoire.

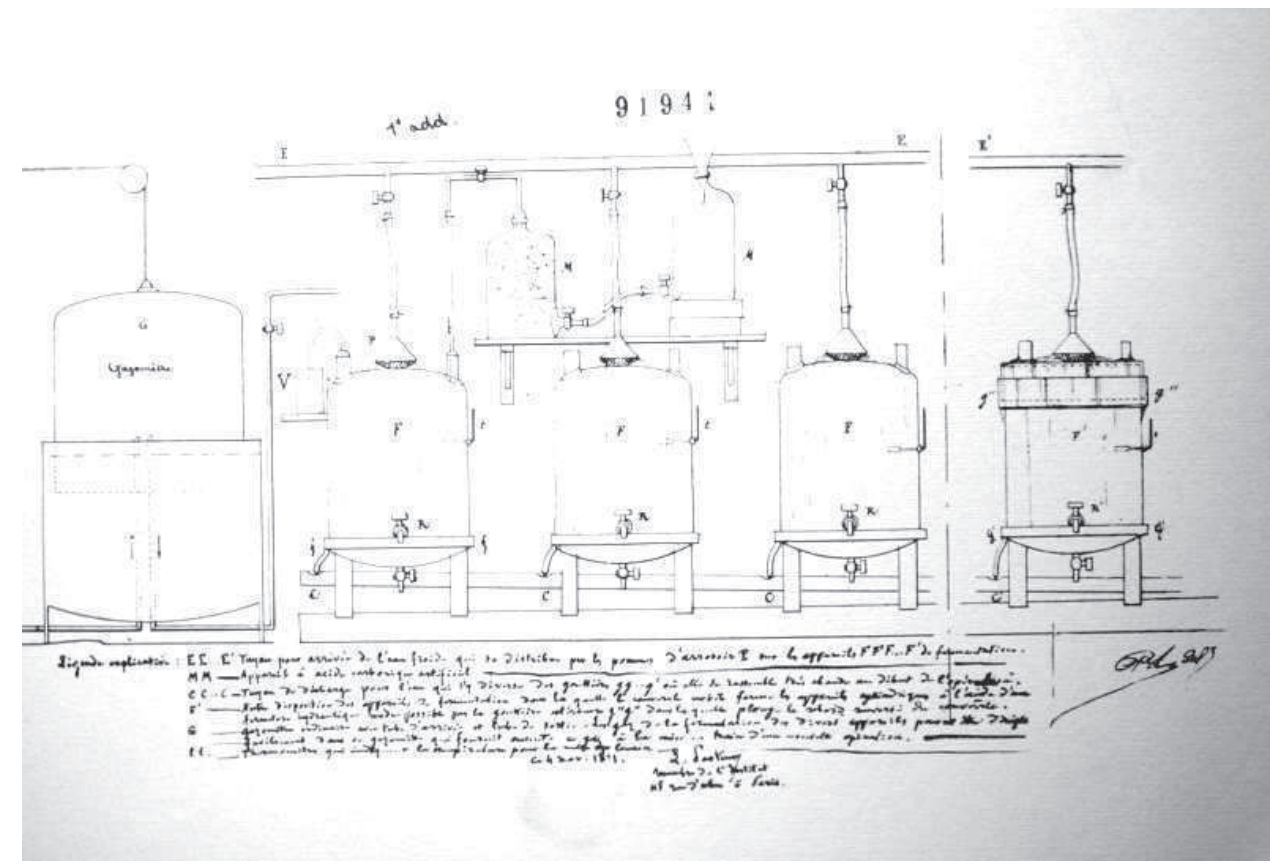

Figure 3: Même tracé à la main, le dessin fait partie de tous les brevets, comme celui de la pasteurisation de la bière dit "de la revanche nationale" par Louis Pasteur. Brevet $n^{\circ}$ 91941 "pour un nouveau procédé de fabrication de la bière » par Louis Pasteur (28-06-1871). Tous les domaines de production sont représentés dans les brevets. Collection S.E.I.N. (bibliothèque S.E.I.N.) 


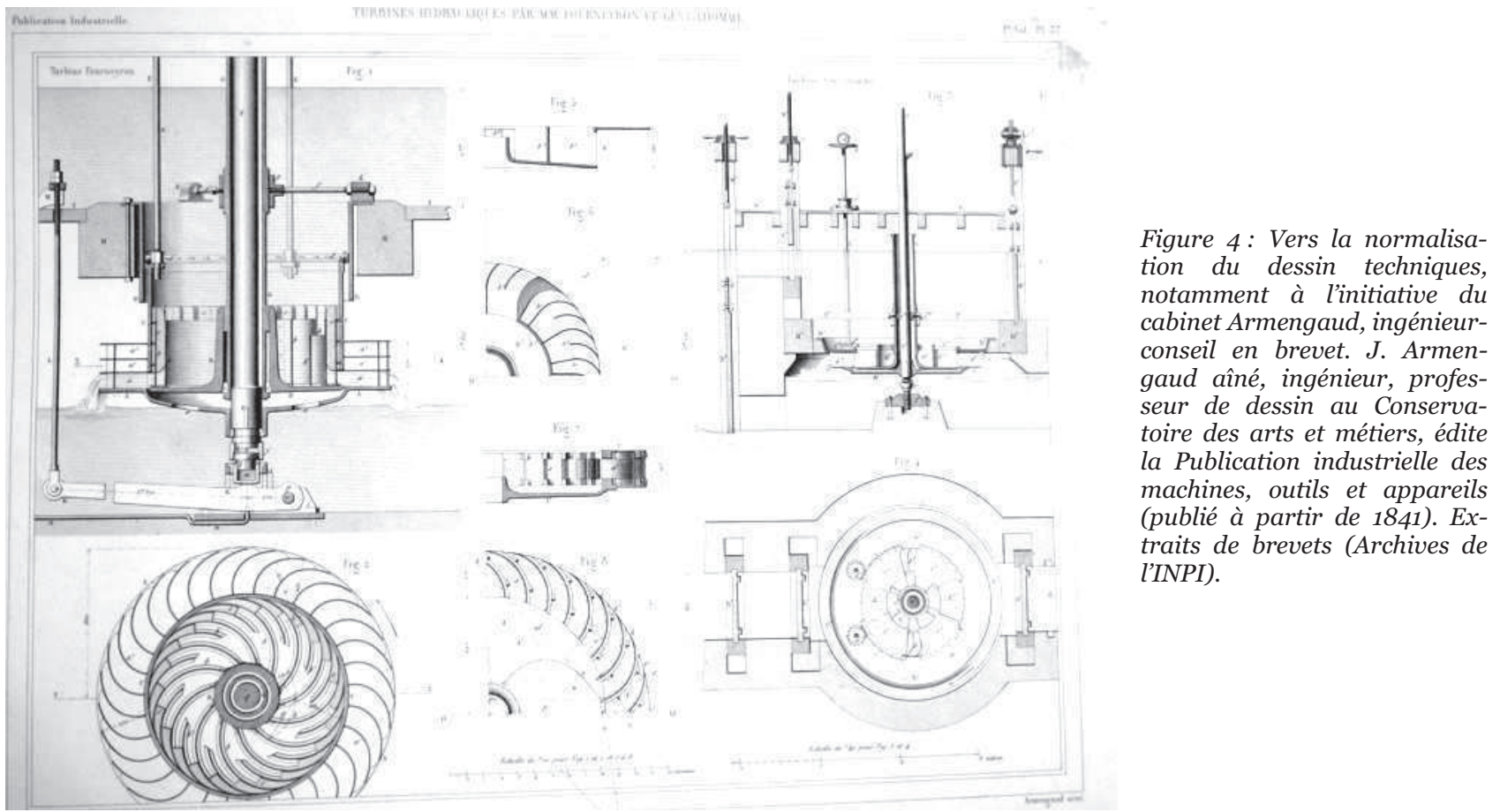

A ce sujet, on ne peut mettre de côté l'intervention des cabinets d'ingénieurs-conseils en brevets pour les dépôts. Ces cabinets, en tant que représentants des inventeurs, rédigent les textes et font les dessins d'accompagnement. Il convient donc de prendre en compte leur intervention dans la lecture des dessins de brevets, puisque la mention du cabinet est toujours indiquée. Leurs dessinateurs, chargés d'illustrer les descriptions, utilisent les normes de graphisme qu'ils ont apprises dans les écoles d'ingénieurs ou de techniciens. Les cabinets en brevets sont apparus au début du XIX siècle. L'un des plus anciens et des plus connus a été le cabinet fondé par Eugène Armengaud (dit l'Aîné). Issu de l'Ecole des arts et métiers de Chalons, devenu ingénieur-conseil, cet ingénieur civil a été aussi professeur de dessin au Conservatoire des arts et métiers. Sous son influence, le dessin technique a été perfectionné et diffusé au moyen d'ouvrages et de périodiques spécialisés pour décrire des objets et des machines (figure 4) 25 .

Un autre problème est aussi à intégrer dans la lecture des dessins. Le brevet n'est pas un article pour une revue de spécialiste. L'inventeur n'étant en général pas disposé à donner trop de détails sur son invention, les dessins sont très schématiques. De plus, les dessins ne sont accompagnés d'aucune échelle quelconque. D'ailleurs, aucune obligation légale ne figure en ce sens.

Un exemple en est donné ici. Il s'agit de la tour inventée par trois ingénieurs civils dans un brevet déposé en 1885. Le plus connu de ces inventeurs est Gustave Eiffel, ingénieur de l'Ecole centrale des arts et manufactures. Il s'agissait de se positionner dans l'appel d'offres qui était lancé dans le cadre de la préparation de l'Exposition universelle de 1889. Il s'agit seulement d'un projet et les détails ne sont nullement fournis. La protection de l'invention était nécessaire vis à vis des concurrents pour le projet d'une tour. Le graphisme du brevet est très éloigné 
de la tour qui sera réellement construite peu après (Figure 5).

Le changement technique peut être perçu au cours d'une même période historique. Deux brevets permettent de la constater : le premier est celui déposé par Clément Ader en 1890 par l'intermédiaire du cabinet Armengaud (figure 6) et le second est le brevet déposé par Rodolph Diesel pour son moteur en 1892, par l'intermédiaire du cabinet Thirion (figure 7). Si les deux inventions sont de grande portée historique, les graphismes sont cependant bien éloignés, alors que dans les deux cas ce sont des dessins d'ingénieurs confirmés. La nature des inventions est la cause de ces grandes différences alors qu'elles sont contemporaines. Les critères de description peuvent donc influer sur le graphisme adopté par les inventeurs.

Après 1902, année à partir de laquelle tous les brevets sont imprimés, le graphisme passe entre les mains de l'Office national de la propriété industrielle, puis de l'INPI, désormais chargés de l'impression des brevets. Le dessin est désormais très schématique, assez simplifié, soumis à des normes, ce qui donne lieu à des représentations très souvent difficiles à déchiffrer si l'on n'est pas un spécialiste du domaine concerné. Des légendes sont toutefois présentes régulièrement avec renvoi vers le texte descriptif de l'invention. Un brevet déposé en 1976 vient illustrer l'état actuel de la représentation des dessins (Figure 8).

\section{Vers une histoire comparée des dessins de brevets}

Pour aller en avant dans la recherche sur les dessins des brevets, abordée ici très schématiquement, il est intéressant de croiser les remarques qui viennent d'être faites avec des travaux sur l'histoire du dessin technique.

Sur ce thème l'ouvrage d'Yves Deforge

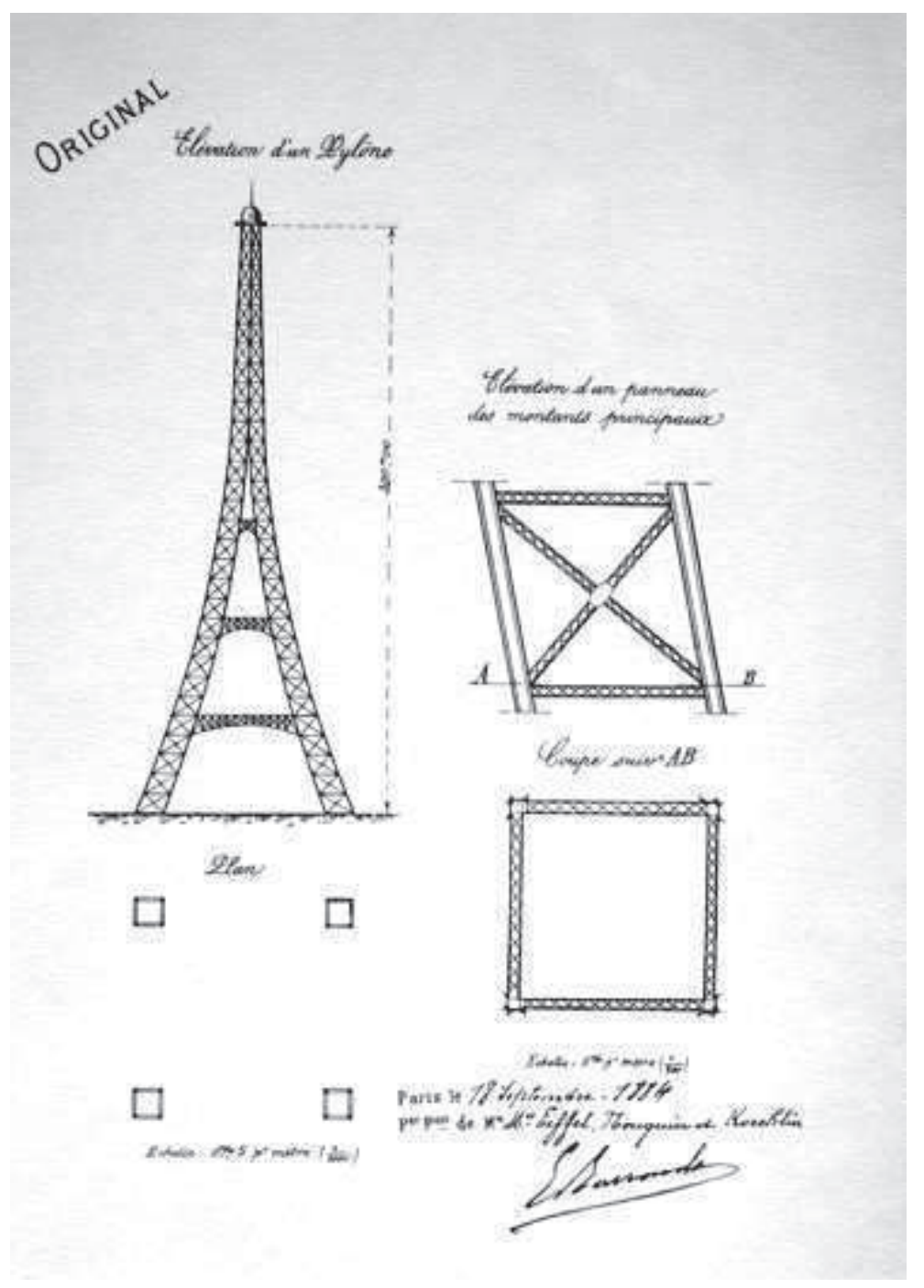

Figure 5: Pour les ingénieurs, comme le centralien Gustave Eiffel, les détails de constructions ne sont pas toujours livrés dans leurs brevets. L'idée seulement ou la protection de l'invention en priorité ? Des ingénieurs qui simplifie à l'extrême leur dessin technique: le cas de la Tour Eiffel. Brevet $n^{\circ} 164364$ de G. Eiffel, E. Nouguier et M. Koechlin, représenté par M. Barrault, pour une "disposition nouvelle permettant de construire des piles et des pylônes métalliques d'une hauteur pouvant dépasser 300 mètres » (30-01-1885). Extraits de brevets (Archives de l'INPI). 
reste un référence ${ }^{26}$. Cet auteur a donné plusieurs clés utiles sur l'histoire du graphisme technique au cours de son évolution progressive depuis le XVIII siècle jusqu'à nos jours. Plus généralement, le dessin technique a fait régulièrement l'objet d'études et d'expositions, comme Madeleine Pinault-Sorensen a pu le souligner avec d'autres observateurs ${ }^{27}$. En ce qui concerne la première moitié du XIX ${ }^{\mathrm{e}}$ siècle, il convient aussi de souligner les travaux d'Alain Mercier menés sur le Portefeuille industriel du Conservatoire national des arts et métiers. Toute une série d'articles posent la question des relations entre les inventeurs et leurs dessins, et de nombreuses machines reproduites dans ces travaux peuvent être comparées à celles des brevets à la même période historique ${ }^{28}$.

Par ailleurs, il convient de prendre en considération la question de la formation au dessin technique en France, comme l'a fait Renaud d'Enfert dans plusieurs études ${ }^{29}$. Enfin, une autre source intéressante ici est celle des dessins techniques parus dans les nombreux ouvrages scientifiques et techniques publiés au cours du XIX ${ }^{\mathrm{e}}$ siècle. Elle est aussi une base extrêmement riche pour toute étude comparative entre images de l'invention et images de la transmission des connaissances. Enfin, le graphisme, sous l'influence de la représentation photographique, doit être signalé.

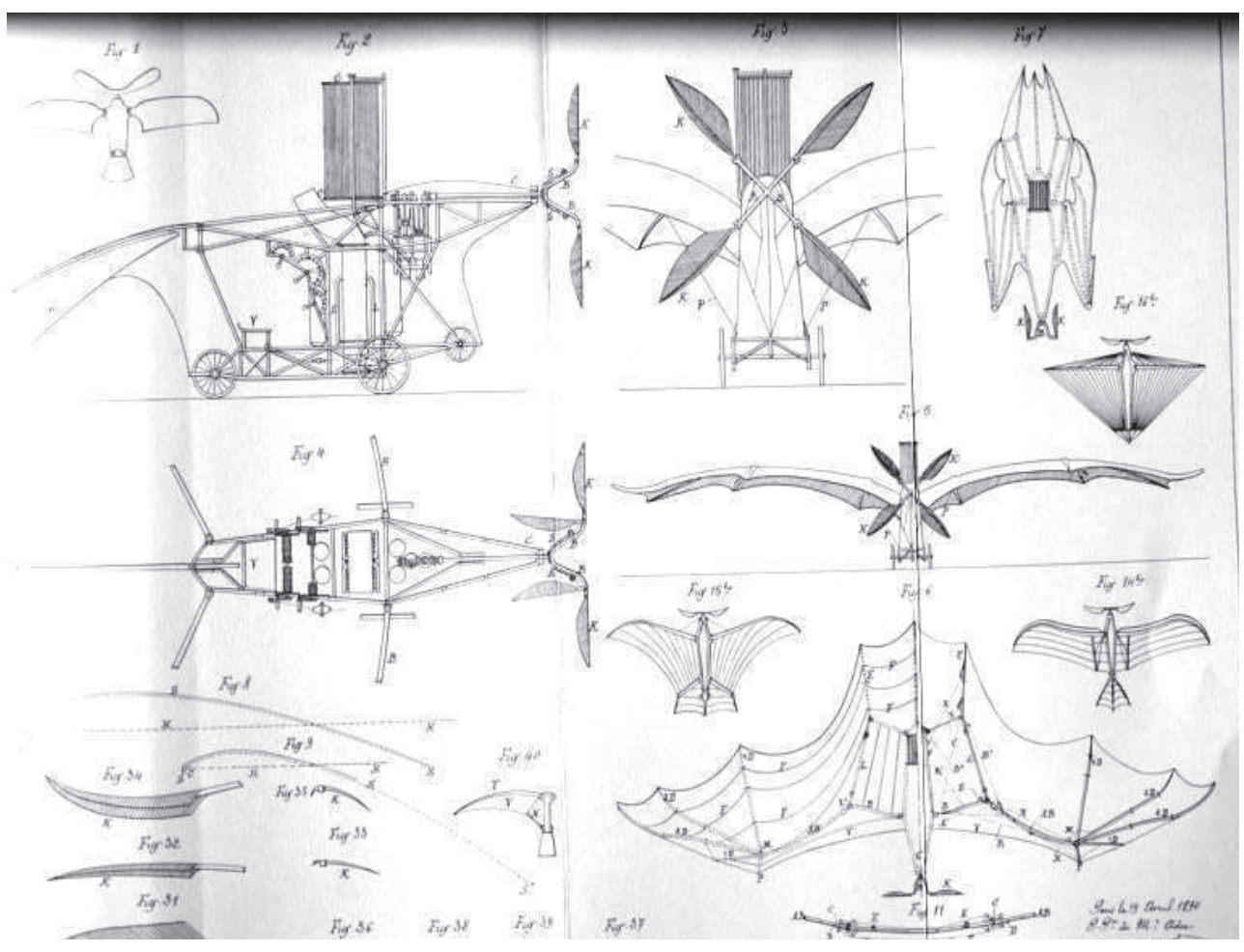

Figure 6: L'adaptation du dessin dans les brevets pour représenter une nouvelle technique peut se poser régulièrement à la fin du siècle. Émergence de nouvelles technologies: représentations adaptées. Le cas de l'aviation. Brevet $n^{\circ} 205155$, déposé par $C$. Ader, représenté par Armengaud jeune, pour un "Appareil ailé pour la navigation aérienne dit " avion 》 » $\left(11-\right.$ O4- $^{-}$ 1880), avec trois certificats d'addition (1891, 1895, 1898). 
La mise en perspective présentée par toutes ces recherches vient enrichir le cas particulier du dessin dans les brevets. La représentation des objets dessinés dans des brevets d'invention constitue, dans cet immense corpus, une intéressante illustration des évolutions que les auteurs cités ici ont décrites ${ }^{30}$.

En conclusion, malgré les multiples aspects qu'il a pris en l'espace d'un siècle, l'ensemble des dessins contenus dans les brevets d'invention constitue pour les historiens un ensemble unique d'archives du dessin technique en France.

Figure 7: Chaque invention nécessite une représentation particulière dans le brevet, mais la complexité des machines n'est pas un obstacle pour les ingénieurs bien entraînés au dessin industriel. Linvention d'un moteur thermique par R. Diesel, une machine motrice innovante. Brevet $n^{\circ} 220903$ pour un "procédé pour produire du travail moteur par la combustion de combustibles » (14-o4 - 1892), avec deux certificats d'addition (1892, 1895). Représenté par les cabinets thirion (1892) et Armengaud jeune (1895). Extraits de brevets (Archives de l'INPI).

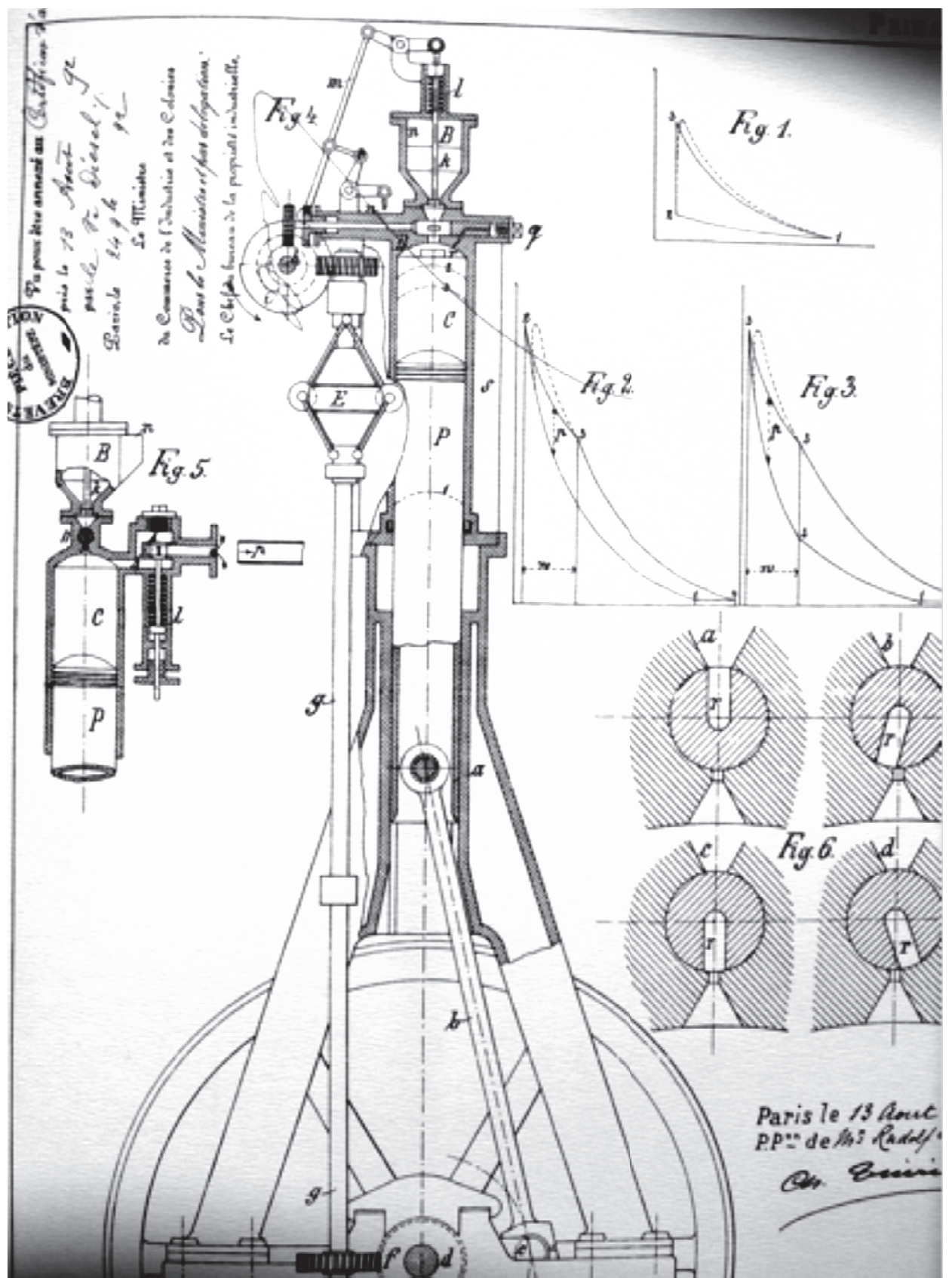




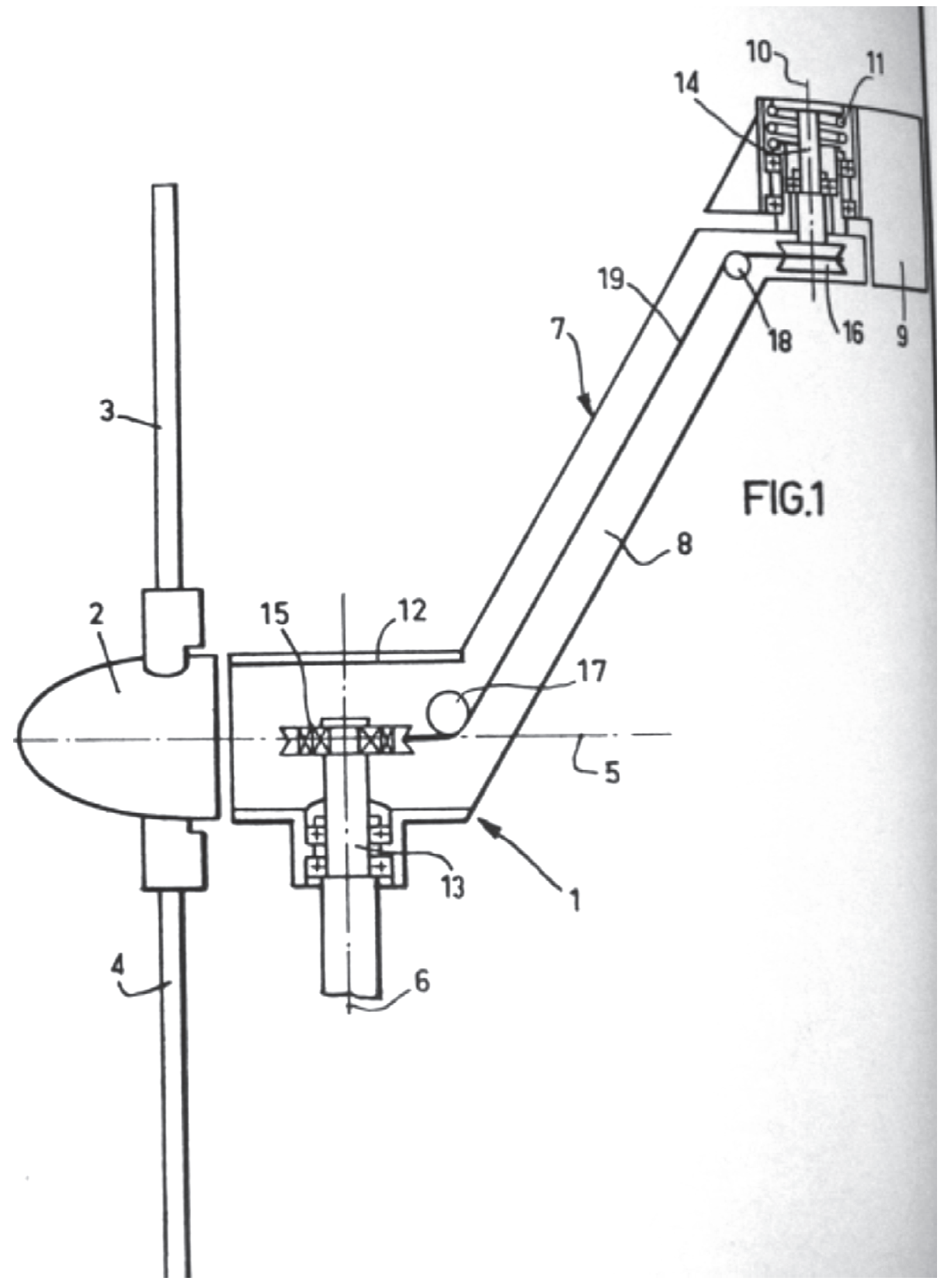

Figure 8: Au XXe siècle, le dessin est désormais très épuré, sans échelle, mais reste référencé pour l'identification de chacun des composants de l'objet inventé. Le brevet contemporain: coup d'œil sur l'énergie éolienne. Aérogénérateur pour la signalisation maritime et aérogénérateur de pompage. Brevet $n^{\circ} 2375649$ de la société Aerowatt (27-12-1972). Extraits de brevets (Archives de l'INPI). 
${ }^{1}$ La partie de ce texte portant sur les aspects historiques de la législation sur les brevets et de leur conservation est une version remaniée d'une communication orale présentée par l'auteur et Valérie Marchal, responsable de la Cellule Archives et Patrimoine de l'INPI, au colloque « La mémoire de la science », tenu en juin 2000 à Paris. L'auteur adresse ses remerciements à Valérie Marchal pour sa contribution.

${ }^{2}$ Pour une vue d'ensemble sur l'utilisation des brevets, voir: Les brevets, leur utilisation en histoire des techniques et de l'économie, Actes Table-ronde CNRS Gif-sur-Yvette, 6-7 déc.1984, C.N.R.S.-I.H.M.C., Paris 1985. Voir aussi : CORCY Marie-Sophie, DEMEULENAERE Christiane et HILAIRE-PEREZ Liliane (dir.), Les archives de l'invention, Toulouse, CNRS-U. Toulouse Le Mirail, 2006. Un colloque sur le même sujet s'est tenu en octobre 2001 (actes non publiés à ce jour).

3 Brevets et Révolution, 1791-1805, Catalogue d'exposition. Paris : Institut National de la Propriété Industrielle, 1989

4 FRESSONNET Pierre, "Le brevet d'invention en France », dans Eureka 83, Un siècle d'inventions françaises,. Paris : C.C.I., INPI, 1983, pp.8-15; pour une perspective générale, voir : PLASSERAUD Yves et SAVIGNON Francois, L'Etat et l'invention. Histoire des brevets. Paris : Documentation Française, 1986.

5 Resté à cette adresse jusqu'en 2012, l'INPI vient de s'installer récemment à l'adresse suivante: INPI, 15 rue des Minimes, CS50001, 92677 Courbevoie Cedex

${ }^{6}$ MARCHAL Valérie, Communication orale à l'Ecole nationale du patrimoine, 1999.

7 L'INPI a créé à cet effet la Cellule Archives et Patrimoine (Département de la Documentation et de l'Information) en 1997, qui a entrepris des inventaires de l'ensemble des fonds patrimoniaux dont l'institut est dépositaire. À partir de ces travaux, une politique globale de conservation et de gestion de ces fonds a été élaborée dans le cadre d'une convention avec la Direction des Archives de France.

${ }^{8}$ Code de la Propriété intellectuelle, article L. 611-10.

${ }_{9}^{9}$ Code de la Propriété intellectuelle, article L. 611-11.

${ }^{10}$ La loi française du 1er juillet 1992 institue le Code de la Propriété Intellectuelle qui regroupe les lois sur le droit d'auteur, les dessins et modèles, les brevets d'invention, le secret de fabrique, les produits semi-conducteurs, les obtentions végétales, les marques et les appellations d'origine.

${ }^{11}$ Arrêté du 5 Vendémiaire an IX (27 septembre 1800).

12 Ibid.. C'est à dire "sans garantie du gouvernement », signalé par le sigle SGDG bien connu.

${ }_{13}$ Pour plus de détails, consulter l'ouvrage d'EMPTOZ Gérard et MARCHAL Valérie, Aux sources de la propriété industrielle.
Guide des archives de l'INPI, Paris, Institut national de la propriété industrielle, 2002, $252 \mathrm{p}$ (en vente à l'INPI).

${ }^{14}$ Certains dossiers de dépôt de brevets sont conservés dans les Archives départementales (dépôts provenant des Préfectures où les inventeurs avaient demandé un brevet), mais ces collections restent purement locales.

15 Voir sur le site de l'INPI la rubrique "Brevets anciens $19^{\mathrm{e}}$ siècle».

16 http://www.inpi.fr/fr/l-inpi/nos-ressources-documentaires. html

${ }^{17}$ Lorsqu'elle sera terminée, la base couvrira la période de 1791 à 1901, soit 330.240 brevets.

18 Pour plus de détails, consulter le site Internet de l'INPI : http://www.inpi.fr/fr/services-et-prestations/bases-de-donnees-gratuites.html . Des bases informatisées spécialisées couvrent différents domaines à partir de 1968 (plusieurs sites dont l'INPI, et des bases sur des documentations distinctes). Se reporter aussi au Guide des archives de l'INPI (op. cit .), p.104-105.

19 Suite à une étude conjointe de l'INPI avec la Société d'encouragement pour l'industrie nationale, réalisée en 1967 sous la direction de Micheline Monka (INPI).

${ }^{20}$ EMPTOZ Gérard et MARCHAL Valérie, 2002, op.cit.

${ }^{21}$ Dans cet ouvrage abondamment illustré, on trouve des informations sur les principaux aspects de la propriété industrielle : brevets, marques, dessins et modèles, avec des documents historiques, des textes législatifs, des statistiques, etc.

${ }^{22}$ Cette partie correspond à une version remaniée et complétée de l'intervention de l'auteur au séminaire présenté le 20 octobre 2010 à l'université Paris 1. Panthéon-Sorbonne L'ensemble des images qui y ont été présentées est accessible sur le site: epi.univ-paris1.fr/.../o/fiche.../\&RH=epi-O3O-s2\&RF=epi-o3os2

${ }^{23}$ Voir les travaux de JOBERT Philippe, en particulier « JeanBaptiste Mollerat ((1772-1855). Le prix de l'innovation », dans LAMARD Pierre et STOSKOPF Nicolas (dir.), L'industrie chimique en question, Paris, 2010, p. 29-39 ; sur la carbonisation du bois : « Jean-Baptiste Mollerat (1772-1855). Un pionnier de la chimie française ", Histoire, Economie et Sociétés, 1991, p. 249268.

24 DEFORGE Yves, Le graphisme technique, son histoire et son enseignement, Paris, Champ Vallon, 1981

${ }^{25}$ Voir l'étude de PEYRE Philippe, "Les Armengaud, la Petite Ecole et le développement de l'innovation », Cahiers d'histoire du CNAM, vol.4, juillet 1994, p .93-142

${ }^{26}$ DEFORGE Yves, 1981, op. cit.

27 PINAULT-SORENSEN Madeleine, «Le dessin, témoin de l'innovation technique ", dans HILAIRE-PEREZ L. et GARÇON 
A-F. (dir.), Les chemins de la nouveauté. Innover, inventer au regard de l'histoire, Paris, Editons du CTHS, 2003, p. 343-360.

${ }^{28}$ Voir notamment MERCIER Alain, « Portefeuille industriel : Le Portefeuille de Vaucanson », La Revue, ${ }^{\circ} 19$, mars 1995. Mention est faite des brevets déposés en France jusqu'en 1817. Cet auteur a publié régulièrement toute une série d'articles sur les dessins conservés au CNAM dans le Portefeuille industriel dans les numéros de La Revue du Musée des arts et métiers, à partir de 1992. Par ailleurs, le Bulletin de la Société d'encouragement pour lindustrie nationale a régulièrement publié, à partir de 1802, des planches hors texte dont le graphisme technique est une source historique d'un grand intérêt pour la présente étude.

${ }^{29}$ Voir en particulier d'ENFERT Renaud, L'enseignement $d u$ dessin en France. Figure humaine et dessin géométrique (17501850), Paris, Belin, 2003. Parmi d'autres travaux de l'auteur sur le dessin technique : "L'enseignement du dessin au XIXe siècle : une affaire d'État? ", dans POULOT D., PIRE J-M. et BONNET A., L'éducation artistique en France. Du modèle académique et scolaire aux pratiques actuelles, XIX ${ }^{e}-X X I^{e}$ siècles, Rennes, Presses universitaires de Rennes, 2010, pp. 285-295.

3o Pour compléter une recherche sur le sujet, on peut trouver dans le Guide des archives de l'INPI de multiples illustrations, extraites de brevets originaux, qui permettent de suivre l'évolution des dessins des brevets d'invention. 